\title{
Barreras para la movilización temprana en la Unidad de Cuidados Intensivos en México
}

\section{Barriers for early mobilization in the Intensive Care Unit in Mexico}

\author{
Miguel Á. Martínez-Camacho", Orlando R. Pérez-Nieto², Manuel A. Guerrero-Gutiérrez ${ }^{3 *}$, \\ Eder I. Zamarrón-López ${ }^{4}$, Raúl Soriano-Orozco ${ }^{5}$, Ernesto Deloya-Tomas ${ }^{6}$, Jesús S. Sánchez-Díaz, \\ Luis A. Morgado-Villaseñor ${ }^{8}$ y Diego Escarramán-Martínez ${ }^{9}$
}

${ }^{1}$ Departamento de Fisioterapia, Hospital General de San Juan del Río, Querétaro, Qro.; ${ }^{2}$ Unidad de Cuidados Intensivos. Hospital General de San Juan del Río, Querétaro, Qro.; ${ }^{3}$ Departamento de Anestesiología, Centro Médico Nacional La Raza, IMSS, Ciudad de México; ${ }^{4}$ Unidad de Cuidados Intensivos, Hospital CEMAIN, Tampico, Tamps.; ${ }^{5}$ Unidad de Cuidados Intensivos, Hospital de Alta Especialidad T1, IMSS, León, Gto.; ${ }^{6}$ Unidad de Cuidados Intensivos, Hospital General de San Juan del Río, Querétaro, Qro.; ${ }^{7}$ Unidad de Cuidados Intensivos, UMAE 189 IMSS, Veracruz, Ver.; ${ }^{8}$ Unidad de Cuidados Intensivos, UMAE, IMSS, Torreón, Coah.; ${ }^{9}$ Departamento de Anestesiología, Centro Médico Nacional La Raza, IMSS, Ciudad de México. México

\section{Resumen}

Introducción: El paciente de la Unidad de Cuidados Intensivos (UCI) presenta una serie de complicaciones funcionales derivadas del proceso de hospitalización. La inmovilidad prolongada, ventilación mecánica, inflamación, sepsis, hiperglucemia, desnutrición, uso de fármacos, entre muchas otras cosas, ponen en riesgo al enfermo de desarrollar morbilidades que complican el manejo en la UCl y pueden generar un mal pronóstico funcional. Objetivo: Describir las principales barreras para la realización de movilización temprana en adultos en la UCI en México. Método: Se encuestó a un total de 102 profesionales de la salud de siete UCI de adultos en México para describir las principales barreras para la movilización temprana. Resultados: El personal involucrado en el manejo del paciente crítico no ha recibido capacitación para la realización de movilización temprana, la funcionalidad no es un tema que constantemente se discuta como parte del manejo del paciente crítico. El $0.98 \%$ del personal encuestado son fisioterapeutas. Conclusión: Se necesitan medidas institucionales para el cambio de paradigma respecto a la funcionalidad del paciente. Es necesario hacer campañas y educación a gran escala en materia de funcionalidad, prevención de secuelas y fisioterapia en el paciente crítico.

Palabras clave: Movilización temprana. Barreras para la movilización. Fisioterapia.

\section{Abstract}

Introduction: The patient in the Intensive Care Unit (ICU) presents a series of functional complications derived from the hospitalization process. Prolonged immobility, mechanical ventilation, inflammation, sepsis, hyperglycemia, malnutrition, drug use, among many other things puts the patient at risk of developing morbidities that complicate ICU management and can generate a poor functional prognosis. Objective: Describe the main barriers to the realization of early mobilization in adults in the intensive care unit in Mexico. Method: A total of 102 health professionals from 7 Adult Intensive Care units in Mexico were surveyed to describe the main barriers to early mobilization. Results: The personnel involved in the management of

Correspondencia:

*Manuel A. Guerrero-Gutiérrez

E-mail: manuelguerreromd@gmail.com
Disponible en internet: 19-06-2020 Rev Hosp Jua Mex. 2020;87(2):58-64

www.revistahospitaljuarez.com 1405-9622/@ 2020 Sociedad Médico-Quirúrgica del Hospital Juárez de México, A.C. Publicado por Permanyer. Este es un artículo open access bajo la licencia CC BY-NC-ND (http://creativecommons.org/licenses/by-nc-nd/4.0/). 
the critical patient have not received training to carry out early mobilization, functionality is not an issue that is constantly discussed as part of the management of the critical patient. $0.98 \%$ of the staff surveyed are physiotherapists. Conclusion: Institutional measures are needed to change the paradigm regarding patient functionality. Campaigns and education on a large scale in terms of functionality, prevention of sequelae and physiotherapy in the critical patient are necessary.

Key words: Early mobilization. Barriers to mobilization. Physiotherapy.

\section{Introducción}

El paciente de la Unidad de Cuidados Intensivos (UCI) presenta una serie de complicaciones funcionales derivadas del proceso de hospitalización. La inmovilidad prolongada, ventilación mecánica, inflamación, sepsis, hiperglucemia, desnutrición, uso de fármacos, entre muchas otras cosas, ponen en riesgo al enfermo de desarrollar morbilidades que complican el manejo en la $\mathrm{UCl}$ y pueden generar un mal pronóstico funcional. La debilidad adquirida en la $\mathrm{UCl}$, la neumonía asociada a ventilación mecánica, las úlceras por presión y la trombosis venosa profunda son situaciones que a menudo se observan en el paciente ingresado en la $\mathrm{UCl}$ y que pueden evitarse $\mathrm{e}^{1-11}$.

Una de las estrategias para la prevención de complicaciones en la $\mathrm{UCl}$ es la movilización temprana, la cual es definida como la realización de ejercicio físico a partir del segundo a quinto día después del inicio de la patología crítica o lesión ${ }^{3,6}$. La movilización temprana es una intervención segura en pacientes bien seleccionados, y la mayoría de complicaciones son menores y de resolución sencilla ${ }^{6,12,13}$. Una adecuada evaluación neurológica, hemodinámica y respiratoria minimizará el riesgo de la intervención ${ }^{6,13,14}$. La movilización temprana es una de las estrategias mencionadas en Guías sobre la prevención y manejo del dolor, agitación/sedación, delirium, inmovilidad y alteraciones del sueño en pacientes adultos en la UCl, propuestas por la Sociedad de Medicina de Cuidados Críticos ${ }^{1}$.

Recientes metaanálisis arrojan como resultados, que la movilización temprana es una estrategia que reduce la prevalencia de debilidad adquirida en la UCl, la neumonía asociada a ventilación mecánica, las escaras por presión y la trombosis venosa profunda ${ }^{15,16}$, además de mejorar la fuerza muscular y el grado de independencia. También hay una reducción en los días de estancia en la $\mathrm{UCl}$ y el hospital, lo cual además tiene un impacto financiero ${ }^{7}$. Mejía, et al. describen un aumento de la fuerza muscular y una reducción del grado de dependencia en pacientes sometidos a un programa de movilización temprana en un hospital de segundo nivel en población mexicana ${ }^{8}$.
Las barreras para la movilización son diversas, desde situaciones relacionadas con la seguridad del paciente o ausencia de fisioterapeutas en la UCl hasta el desconocimiento del procedimiento por parte del personal. Hoyer, et al. realizaron un estudio para conocer los principales obstáculos para la movilización temprana y encontraron que el personal piensa que movilizar a los pacientes aumenta la carga del personal de enfermería. Por otro lado, Jones, et al. describieron las principales barreras para la movilización temprana en una $\mathrm{UCI}$ pediátrica en la ciudad mexicana de Querétaro, y reportaron que la principal limitante es la falta de material y mobiliario. En este estudio, la movilización temprana en la población pediátrica parece ser bien aceptada, los profesionales conocen los beneficios de la intervención y están de acuerdo en su realización $n^{3,17}$.

Existen pocos estudios sobre movilización temprana en población mexicana, y no hay datos sobre las barreras para su realización en la población adulta, pacientes en los que existe el mayor grado de evidencia de beneficio. El objetivo de este estudio es describir las principales barreras para la realización de movilización temprana en adultos en la $\mathrm{UCl}$ en México.

\section{Material y método}

Tipo de estudio: multicéntrico observacional y transversal.

En el periodo de julio a agosto de 2019 se aplicó un cuestionario a personal de salud involucrado en el manejo del paciente crítico en la $\mathrm{UCl}$ de adultos. El cuestionario se envió vía electrónica a las diversas unidades que quisieron participar en el estudio y posteriormente se devolvió contestado al investigador.

Se utilizó un instrumento elaborado por Hoyer, et al. en 2015 y validado por Jones, et al. en 2019 para población mexicana. Dicho cuestionario consta de 26 ítems, divididos en 3 subdominios: conocimiento, actitud y comportamiento. Se verificó la fiabilidad por medio de alfa de Cronbach.

Se realizó el análisis estadístico por medio del software IBM SPSS Versión 21, y se obtuvieron porcentajes y frecuencias para variables categóricas y medidas de tendencia central para variables numéricas. 


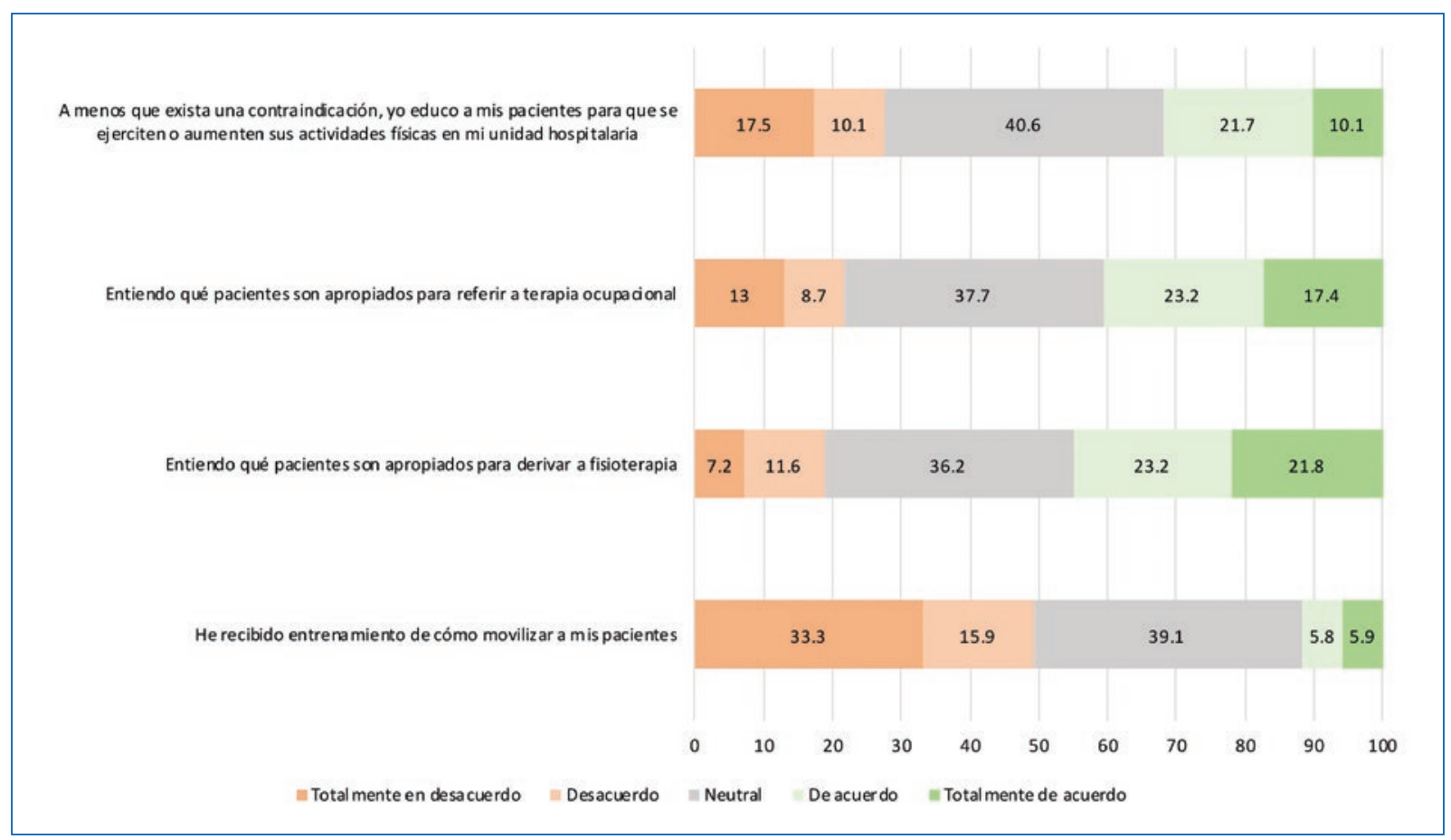

Figura 1. Porcentaje de respuestas para el subdominio conocimiento $(n=102)$.

Tabla 1. Resultados del subdominio de conocimiento

\begin{tabular}{|c|c|c|c|c|c|c|c|}
\hline N. ${ }^{\circ}$ de ítem & Ítem & $1(\%)$ & $2(\%)$ & $3(\%)$ & $4(\%)$ & $5(\%)$ & Media (DE) \\
\hline 2 & $\begin{array}{l}\text { He recibido entrenamiento de cómo movilizar a mis } \\
\text { pacientes }\end{array}$ & 33.3 & 15.9 & 39.1 & 5.8 & 5.9 & $2.3(1.0)$ \\
\hline 5 & $\begin{array}{l}\text { Entiendo qué pacientes son apropiados para derivar a } \\
\text { fisioterapia }\end{array}$ & 7.2 & 11.6 & 36.2 & 23.2 & 21.8 & $3.4(1.2)$ \\
\hline 6 & $\begin{array}{l}\text { Entiendo qué pacientes son apropiados para referir a } \\
\text { terapia ocupacional }\end{array}$ & 13.0 & 8.7 & 37.7 & 23.2 & 17.4 & $3.2(1.2)$ \\
\hline 25 & $\begin{array}{l}\text { A menos que exista una contraindicación, yo educo a mis } \\
\text { pacientes para que se ejerciten o aumenten sus actividades } \\
\text { físicas en mi unidad hospitalaria }\end{array}$ & 17.5 & 10.1 & 40.6 & 21.7 & 10.1 & $3.8(1.1)$ \\
\hline
\end{tabular}

\section{Resultados}

Se encuestó a un total de 102 profesionales de la salud de siete $\mathrm{UCl}$ de adultos en México. De ellos, el $52.94 \%$ fue personal de enfermería, el $46.08 \%$ médicos y el $0.98 \%$ fisioterapeutas, con una media de experiencia total en UCl de $5.4 \pm 4.5$ años. Los estados donde se realizó el cuestionario fueron: Querétaro, Guanajuato, Puebla, Veracruz y Ciudad de México.

Dentro de las barreras más importantes para la movilización temprana se encontraron la ausencia de capacitación para su realización, la percepción de que la movilización temprana aumenta la carga de trabajo del personal de enfermería, una inadecuada relación enfermero-paciente (número de pacientes por cada enfermero), la ausencia de indicación médica y la ausencia de discusión sobre la funcionalidad física del enfermo en la UCI.

Sin embargo, se observaron una serie de facilitadores, como la percepción de que los pacientes no están tan críticos como para no poder ser movilizados, que no se considera que muchos pacientes tengan contraindicaciones para poder ser movilizados y que el 
Tabla 2. Resultados del subdominio de actitud

\begin{tabular}{|c|c|c|c|c|c|c|c|}
\hline N. ${ }^{0}$ de ítem & Ítem & $1(\%)$ & $2(\%)$ & $3(\%)$ & $4(\%)$ & $5(\%)$ & Media (DE) \\
\hline 1 & Mis pacientes están demasiado enfermos para ser movilizados & 37.7 & 33.3 & 18.8 & 8.7 & 1.4 & $2.0(1.0)$ \\
\hline 3 & $\begin{array}{l}\text { Incrementar la movilización de mis pacientes será dañino } \\
\text { para ellos }\end{array}$ & 55.1 & 29.0 & 11.6 & 4.3 & 0.0 & $1.6(0.8)$ \\
\hline 4 & $\begin{array}{l}\text { Un fisioterapeuta o terapeuta ocupacional debe ser el } \\
\text { principal proveedor de la movilización de mis pacientes }\end{array}$ & 14.6 & 25.6 & 35.8 & 17.2 & 6.8 & $2.7(1.0))$ \\
\hline 12 & $\begin{array}{l}\text { Aumentar la movilización de los pacientes generará más } \\
\text { carga de trabajo a enfermería }\end{array}$ & 36.3 & 11.6 & 18.8 & 17.4 & 15.9 & $2.6(1.5)$ \\
\hline 13 & $\begin{array}{l}\text { Aumentar la movilización de los pacientes generará más } \\
\text { carga de trabajo a los fisioterapeutas o terapeutas } \\
\text { ocupacionales }\end{array}$ & 50.8 & 14.5 & 21.7 & 10.1 & 2.9 & $2.0(1.1)$ \\
\hline 18 & $\begin{array}{l}\text { Creo que los pacientes que están siendo movilizados al } \\
\text { menos dos veces al día tienen un mejor egreso hospitalario }\end{array}$ & 2.9 & 5.8 & 44.9 & 15.0 & 31.4 & $3.6(1.0)$ \\
\hline 19 & Desconozco cuándo es seguro movilizar a los pacientes & 45.0 & 24.6 & 15.9 & 8.7 & 5.8 & $2.0(1.2)$ \\
\hline 21 & $\begin{array}{l}\text { No siento confianza con mi habilidad de movilizar a mis } \\
\text { pacientes }\end{array}$ & 40.7 & 33.3 & 13.0 & 7.2 & 5.8 & $2.0(1.1)$ \\
\hline 26 & $\begin{array}{l}\text { Mis pacientes tienen tiempo en su día de ser movilizados } \\
\text { al menos tres veces al día }\end{array}$ & 8.7 & 13.0 & 37.8 & 21.7 & 18.8 & $3.2(1.1)$ \\
\hline
\end{tabular}

$\mathrm{n}=102$.

1: totalmente en desacuerdo; 2 : en desacuerdo; 3: neutral; 4: de acuerdo; 5: totalmente de acuerdo.

Tabla 3. Resultados del subdominio comportamiento

\begin{tabular}{|c|c|c|c|c|c|c|c|}
\hline $\begin{array}{l}\text { N. }{ }^{\circ} \text { de } \\
\text { ítem }\end{array}$ & Ítem & $1(\%)$ & $2(\%)$ & $3(\%)$ & $4(\%)$ & $5(\%)$ & $\begin{array}{l}\text { Media } \\
\text { (DE) }\end{array}$ \\
\hline 7 & No tenemos el equipo ni el mobiliario para movilizar a los pacientes & 15.9 & 15.9 & 37.7 & 11.6 & 18.8 & $3.0(1.3)$ \\
\hline 8 & $\begin{array}{l}\text { La funcionalidad física de mis pacientes es discutida con regularidad } \\
\text { entre los proveedores de salud del paciente }\end{array}$ & 37.7 & 26.1 & 14.5 & 10.1 & 11.6 & $2.3(1.3)$ \\
\hline 9 & $\begin{array}{l}\text { El personal en relación enfermero-paciente es adecuado para } \\
\text { movilizar pacientes en mi unidad }\end{array}$ & 23.2 & 27.5 & 31.9 & 10.1 & 7.3 & $2.5(1.1)$ \\
\hline 10 & Mis pacientes a menudo tienen contraindicaciones para ser movilizados & 21.7 & 39.2 & 20.3 & 13.0 & 5.8 & $2.4(1.1)$ \\
\hline 11 & $\begin{array}{l}\text { A menos que existan contraindicaciones, mis pacientes son } \\
\text { movilizados al menos una vez por enfermería }\end{array}$ & 24.6 & 14.5 & 40.6 & 8.7 & 11.6 & $2.6(1.2)$ \\
\hline 14 & $\begin{array}{l}\text { El liderazgo de mi departamento apoya fuertemente la movilización } \\
\text { de pacientes }\end{array}$ & 14.5 & 29.1 & 33.3 & 13.0 & 10.1 & $2.7(1.1)$ \\
\hline 15 & $\begin{array}{l}\text { Incrementar la movilización aumenta el riesgo de lesiones en mis } \\
\text { pacientes }\end{array}$ & 56.6 & 27.5 & 14.5 & 0.0 & 1.4 & $1.6(0.8)$ \\
\hline 16 & $\begin{array}{l}\text { Pacientes que pueden ser movilizados usualmente tienen órdenes } \\
\text { médicas para la movilización }\end{array}$ & 18.8 & 37.8 & 31.9 & 4.3 & 7.2 & $2.4(1.0)$ \\
\hline 17 & Mis pacientes se resisten a ser movilizados & 59.4 & 18.9 & 13.0 & 5.8 & 2.9 & $1.7(1.0)$ \\
\hline 20 & $\begin{array}{l}\text { Los familiares de mis pacientes normalmente están interesados en } \\
\text { ayudar a movilizarlos }\end{array}$ & 14.5 & 11.6 & 36.2 & 24.7 & 13.0 & $3.1(1.2)$ \\
\hline 22 & $\begin{array}{l}\text { Yo documento el estado de funcionalidad física de mis pacientes } \\
\text { durante mi turno de trabajo }\end{array}$ & 20.3 & 20.3 & 34.8 & 14.5 & 10.1 & $2.7(1.2)$ \\
\hline 23 & Yo no tengo tiempo de movilizar a mis pacientes durante mi turno & 43.5 & 23.2 & 17.4 & 8.7 & 7.2 & $2.1(1.2)$ \\
\hline 24 & $\begin{array}{l}\text { A menos que exista contraindicación, yo movilizo a mi paciente al } \\
\text { menos } 1 \text { vez al día }\end{array}$ & 20.3 & 14.5 & 40.6 & 14.5 & 10.1 & $2.7(1.2)$ \\
\hline
\end{tabular}

$\mathrm{n}=102$

1: totalmente en desacuerdo; 2: en desacuerdo; 3: neutral; 4: de acuerdo; 5: totalmente de acuerdo. 


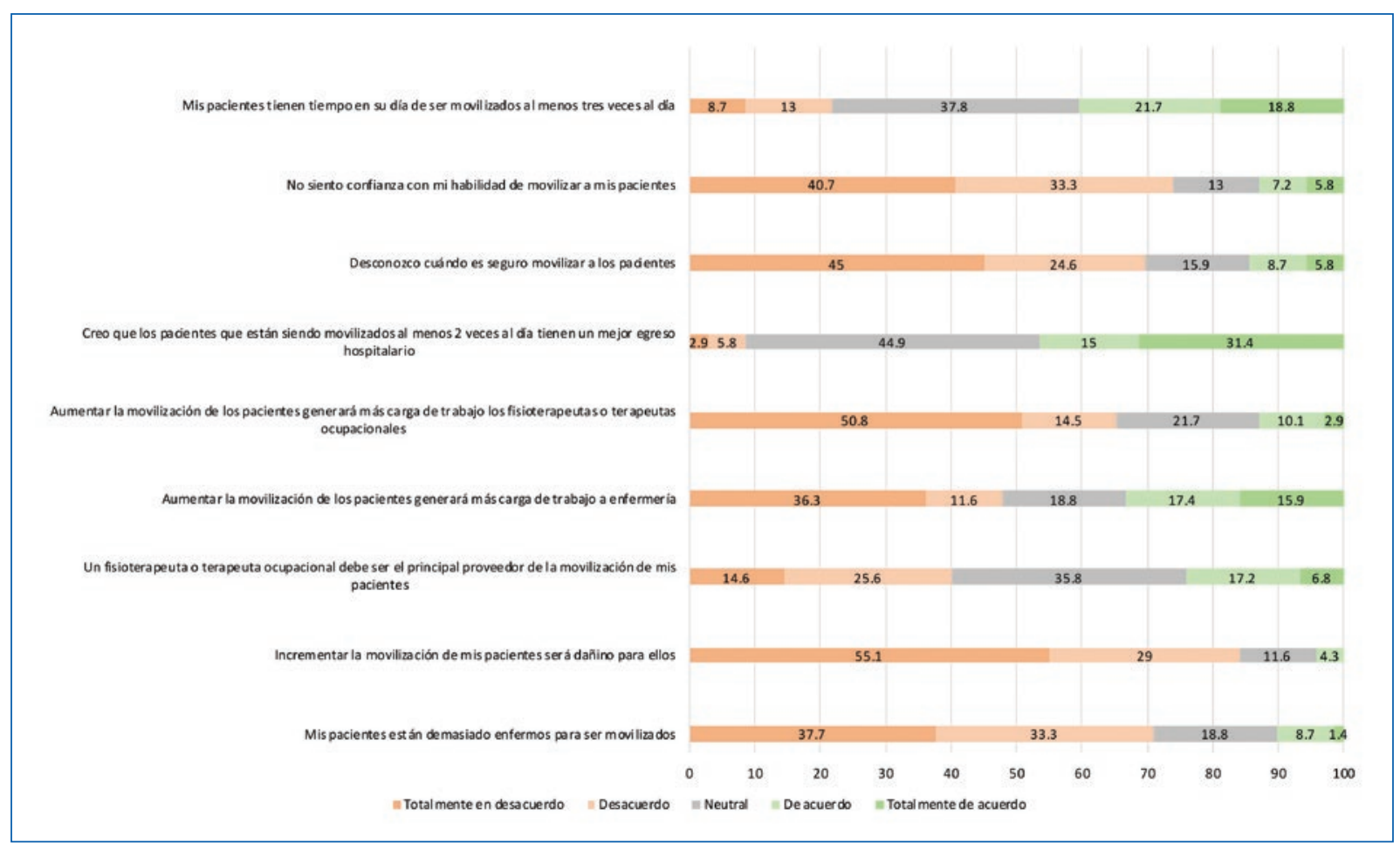

Figura 2. Porcentaje de respuestas para el subdominio actitud $(n=102)$.

procedimiento se ve seguro. El personal considera que puede reconocer cuándo se puede mover a los pacientes y se sienten con la confianza para poder realizarlo (Fig. 1, 2 y 3). Los resultados completos por subescalas se muestran en las tablas 1, 2 y 3 .

De manera adicional se realizó la prueba de fiabilidad del instrumento y se encontró un alfa de Cronbach de 0.80 .

\section{Discusión}

El presente estudio muestra una serie de barreras importantes para la realización de la movilización temprana. En el subdominio de conocimiento, el personal refiere no estar siendo capacitado en materia de movilización del paciente críticamente enfermo, datos similares a los obtenidos en un estudio mexicano publicado en 2019 realizado en una $\mathrm{UCI}$ pediátrica; sin embargo, un estudio americano de 2015 arroja resultados completamente contrarios, el personal en este hospital refiere haber recibido capacitación en movilización temprana ${ }^{3,17}$. En este estudio tampoco se define de manera clara cuál es el rol de los servicios de fisioterapia y terapia ocupacional dentro de las $\mathrm{UCl}$. Esto es interesante, ya que únicamente el $0.98 \%$ de los encuestados fue personal de fisioterapia. Este dato nos hace pensar que la mayoría de los hospitales encuestados no cuenta con servicio de fisioterapia y rehabilitación; este porcentaje es más bajo que el reportado en un estudio para barreras de la movilización en población pediátrica en la Ciudad de Querétaro, con un $3.6 \%^{3}$.

En cuanto al subdominio de actitud parece existir una buena aceptación por parte del personal en el presente estudio, situación similar a lo reportado en la UCI pediátrica. Una diferencia sustancial en el estudio realizado por Hoyer, et al. es la percepción de que la movilización aumenta la carga de trabajo del personal de enfermería, dato contrario a los dos estudios que se han realizado en México ${ }^{3,17}$.

La funcionalidad del paciente parece ser un tema poco discutido en México, lo que se diferencia claramente de lo publicado por otros autores en EE.UU. ${ }^{17}$. Por otro lado, en México, la movilización no es parte de las indicaciones médicas según se observa en este estudio. En otros países, también se ha visto una resistencia importante para la implementación de protocolos de movilización temprana, desde pensar que no es una estrategia prioritaria o por miedo a complicaciones en el paciente mecánicamente ventilado ${ }^{18,19}$. La falta de equipo fue una de las principales barreras para la movilización reportada por Jones, et al., sin embargo, en el 


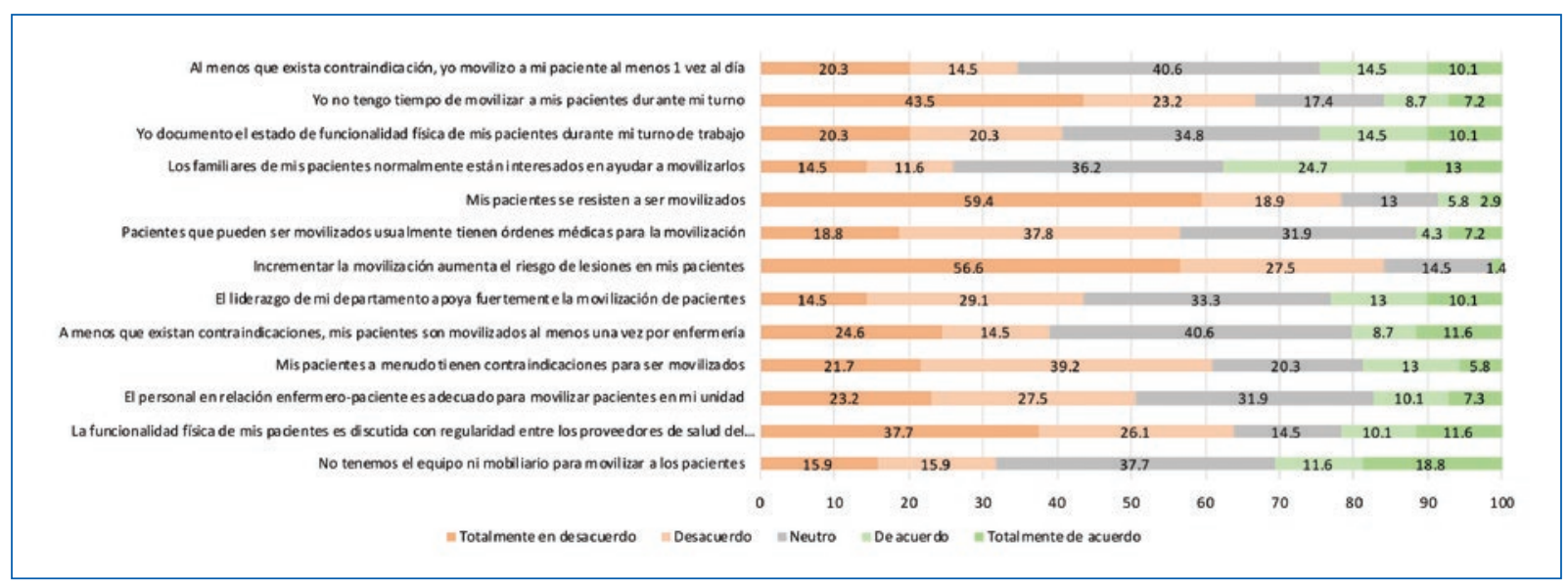

Figura 3. Porcentajes de respuestas para el subdominio comportamiento $(n=102)$.

presente estudio y en el realizado por Hoyer, et al., parece ser una situación neutral ${ }^{3,17}$.

La cultura de la inmovilidad en la $\mathrm{UCl}$ está aún presente en México a pesar de la evidencia que se ha recopilado alrededor del mundo a favor de la movilización temprana. No hay servicios de fisioterapia de manera habitual en los hospitales mexicanos $y$, en caso de haberlos, no atienden a los pacientes desde estadios agudos de la enfermedad crítica. México tiene la necesidad y obligación de capacitarse en materia de movilización temprana para darles dicho beneficio a sus pacientes, además del impacto financiero que pueden tener estas medidas. Es urgente un cambio de paradigma respecto a la funcionalidad del paciente crítico para que se deje de ver como un aspecto secundario y de esta manera, evitar complicaciones que afectan a mediano y largo plazo a las personas que ingresan en una $\mathrm{UCl}$ en México ${ }^{20-23}$.

\section{Conclusión}

En México no hay una adecuada capacitación sobre movilización temprana. Se necesitan medidas institucionales para el cambio de paradigma respecto a la funcionalidad del paciente. Es necesario hacer campañas y educación a gran escala en materia de funcionalidad, prevención de secuelas y fisioterapia en el paciente crítico.

\section{Conflicto de intereses}

Los autores declaran no tener conflicto de intereses alguno.

\section{Financiamiento}

Los autores no recibieron patrocinio para llevar a cabo este artículo.

\section{Responsabilidades éticas}

Protección de personas y animales. Los autores declaran que para esta investigación no se han realizado experimentos en seres humanos ni en animales.

Confidencialidad de los datos. Los autores declaran que en este artículo no aparecen datos de pacientes.

Derecho a la privacidad y consentimiento informado. Los autores declaran que en este artículo no aparecen datos de pacientes.

\section{Bibliografía}

1. J Devlin JW, Skrobik Y, Gélinas C, Needham DM, Slooter AJC, Pandharipande PP. Clinical Practice Guidelines for the Prevention and Management of Pain, Agitation/Sedation, Delirium, Immobility, and Sleep Disruption in Adult Patients in the ICU. Crit Care Med. 2018;46(9): e825-73.

2. Sommers J, Engelbert RH, Dettling-Ihnenfeldt D, Gosselink R, Spronk PE, Nollet F, et al. Physiotherapy in the intensive care unit: an evidence-based, expert driven, practical statement and rehabilitation recommendations. Clin Rehabil. 2015:29(11):1051-63.

3. Jones-Baro RA, Duarte J, Martínez-Camacho MA, Diaz IV, Monjarás SMC. Barreras para la movilizacion temprana en una unidad de cuidados intensivos pediatricos en Mexico. Eur Sci J. 2019;15(21):45-58.

4. Wieske L, Witteveen E, Verhamme C, Dettling-ihnenfeldt DS, Van M. Early Prediction of Intensive Care Unit - Acquired Weakness Using Easily Available Parameters : A Prospective Observational Study. 2014; 9(10):1-8.

5. Choong K. Early Mobilization in Critically III Children: A Systematic Review. J Pediatr [Internet]. 2018; Disponible en: https://doi.org/10.1016/j. jpeds.2018.07.037

6. Cameron S, Ball I, Cepinskas G, Choong K, Doherty J, Ellis CG, et al. Early Mobilization in the Crictical Care Unit: A Review of Adult and Pediatric Literature. J Crit Care [Internet]. 2015; Disponible en: http://dx.doi. org/10.1016/j.jcrc.2015.03.032

7. Zang K, Chen B, Wang M, Chen D, Hui L. The effect of early mobilization in critically ill patients : A meta-analysis. Nurs Crit Care. 2019 Jun 20; 
Rev Hosp Jua Mex. 2020;87(2)

8. Mejía AAC, Martinez NGM, Nieto ORP, Camacho MAM. Movilización Temprana como prevención y tratamiento para la debilidad adquirida en la Unidad de Cuidados Intensivos en pacientes en ventilación mecánica. Experiencia en un hospital de segundo nivel. Eur Sci J. 2018:14(21):19-30.

9. Denehy L, Lanphere J, Needham DM. Ten reasons why ICU patients should be mobilized early. Intensive Care Med [Internet]. 2017; 43(1):86-90.

10. Choong K, Chacon MDP, Walker RG, Timmons GABW, Thabane L, Al-harbi S. In-Bed Mobilization in Critically III Children: A Safety and Feasibility Trial. J Pediatr Intensive Care. 2015;4(4):225-34.

11. Desai S V, Law TJ, Needham DM. Long-term complications of critical care. 2011;39(2):371-9.

12. Care N, Young B, Moyer M, Pino W, Kung D, Zager E, et al. Safety and Feasibility of Early Mobilization in Patients with Subarachnoid Hemorrhage and External Ventricular Drain. Neurocrit Care. 2019;31(1):88-96.

13. Hodgson CL, Stiller K, Needham DM, Tipping CJ, Harrold M, Baldwin CE, et al. Expert consensus and recommendations on safety criteria for active mobilization of mechanically ventilated critically ill adults. Crit Care. 2014;18(6):658

14. Parry SM, Huang M, Needham DM. Evaluating physical functioning in critical care : considerations for clinical practice and research. Critical Care. 2017;21:249.
15. Yue M, Ma ZY, Lei MJ, Cui CY, Jin Y. Early mobilization for mechanically ventilated patients in the intensive care unit: a systematic review and meta-analysis. Frontiers Nurs. 2018;5(4):301-10.

16. Zang $K$, Chen $B$, Wang $M$, Chen $D$, Hui L, Guo S, et al. The effect of early mobilization in critically ill patients: A meta-analysis. Nurs Crit Care. 2019 Jun 20.

17. Hoyer EH, Brotman DJ, Chan KS, Needham DM. Barriers to early mobility of hospitalized general medicine patients: survey development and results. Am J Phys Med Rehabil. 2015;94(4):304-12.

18. Lin F, Phelan S, Chaboyer W, Mitchell M. Early mobilisation of ventilated patients in the intensive care unit: A survey of critical care clinicians in an Australian tertiary hospital. Aust Crit Care. 2020;33(2)130-6.

19. Castro E, Turcinovic M, Platz J, Law I. Early mobilization: changing the mindset. Critical Care Nurse. 2015;35(4): e1-e7.

20. Kress J.P, Hall J.B. ICU-Acquired Weakness and Recovery from Critical Illness. N Engl J Med. 2014;370:1626-35.

21. Hashem MD, Nelliot A, Needham DM. Early Mobilization and Rehabilitation in the ICU: Moving Back to the Future. Respir Care 2016:61(7):971-9.

22. Hermans G, Van der Berghe $G$. Clinical review: intensive care unit acquired weakness. Critical Care. 2015;19:274.

23. Intiso D. ICU-acquired weakness: should medical sovereignty belong to any specialist? Intiso Critical Care. 2018;22:1 